\title{
PREVALÊNCIA DE TRANSTORNOS ALIMENTARES EM ADOLESCENTES PRATICANTES DE BALLET CLÁSSICO
}

\author{
Daniel Vicentini de Oliveira, Centro Universitário de Maringá - CESUMAR, Maringá, \\ Paraná-Brasil \\ Kerolen de Mello Alves, Centro Universitário de Maringá - CESUMAR, Maringá, Paraná \\ - Brasil \\ Carmen Patrícia Barbosa, Centro Universitário de Maringá - CESUMAR, Maringá, \\ Paraná-Brasil
}

\section{RESUMO}

As sociedades ocidentais contemporâneas vivem sob o ideal da magreza e da boa forma física, cujo padrão se impõe especialmente para as mulheres, nas quais a aparência física representa uma importante medida de valor pessoal. Com base nesta preocupação foi realizado este trabalho que tem como objetivo avaliar a prevalência de bulimia e anorexia enquanto transtornos alimentares apresentados por adolescentes praticantes de ballet clássico. Trata-se de uma pesquisa do tipo descritivo, cuja população compreendeu 50 adolescentes do sexo feminino, com idades entre 12 a 18 anos, praticantes de ballet clássico de três academias situadas em Maringá-PR. A coleta de dados foi realizada pela própria pesquisadora antes das aulas de ballet de cada estabelecimento, por meio dos testes que avaliam a presença de sintomas relacionados a transtornos alimentares. De acordo com o questionário BITE, $80 \%$ da população avaliada apresentaram nível baixo de incidência de sintomas de bulimia nervosa encontrando-se dentro dos limites de anormalidade. Para a anorexia nervosa, o valor alto foi verificado em uma das academias pesquisadas observando-se a prevalência de sintomatologia anoréxica. Esses resultados apontam que é importante que o professor de educação física aborde em suas aulas temas como a bulimia e anorexia nervosa, a fim de conscientizar, prevenir e controlar possíveis transtornos alimentares principalmente em adolescentes praticantes de atividades esportivas.

Palavras-Chave: Adolescência; Anorexia; Bulimia; Dança.

\section{PREVALENCE OF EATING DISORDERS ON TEENAGERS PRACTITIONERS OF CLASSICAL BALLET}

\begin{abstract}
The contemporary western societies live under the ideal of thinness and fitness, whose standard is needed especially for women, in which physical appearance is an important measure of worth. Based on this concern was made that this work is to evaluate the prevalence of anorexia and bulimia eating disorders as teenagers presented by practitioners of classical ballet. This is a descriptive research, whose study population comprised 50 female teenagers aged 12-18 years, practitioners of classical ballet in three academies located in Maringá-PR. Data collection was performed by the researcher before the ballet classes in each establishment, by means of tests that assess the presence of symptoms

Conexões: revista da Faculdade de Educação Física da UNICAMP, Campinas, v. 11, n. 1, p. 171-187, jan../mar. 2013. 171 ISSN: 1983-9030
\end{abstract}


related to eating disorders. According to BITE, $80 \%$ of the population tested showed low incidence of symptoms of BN lying within the limits of abnormality. For nervous anorexia, the high value was observed in one of the search academies observing the prevalence of anorexic symptoms. These results indicate that it is important that the teacher of physical education in their classes address themes such as bulimia and nervous anorexia, in order to educate, prevent and control possible eating disorders especially in teenagers who practice sports.

Key-Words: Adolescence; Anorexia; Bulimia; Dance. 


\section{INTRODUÇÃO}

As sociedades ocidentais contemporâneas vivem sob o ideal da magreza e da boa forma física, cujo padrão se impõe especialmente às mulheres, nas quais a aparência física representa uma importante medida de valor pessoal.

O impacto desse padrão no comportamento revela-se no desejo generalizado de um corpo mais magro. A discrepância entre o peso real e o ideal leva a um estado de constante insatisfação com o próprio corpo e as dietas para perder peso tornam-se frequentes fazendo surgir um campo fértil para o desenvolvimento dos transtornos alimentares. ${ }^{1}$

Alguns traços presentes nas crianças em idade escolar são considerados fatores de risco para o surgimento dos transtornos alimentares na adolescência os quais devem servir de alerta ao profissional de saúde que atende essa população. Em algumas populações específicas tais traços são mais marcantes. Isto acontece, por exemplo, em profissões que exigem leveza para melhor desempenho (como ginastas, jockeis, patinadoras, bailarinas). ${ }^{2}$

O presente trabalho teve como objetivo geral avaliar a prevalência de transtornos alimentares, como a anorexia nervosa (AN) e bulimia nervosa (BN), apresentados por adolescentes do sexo feminino praticantes de ballet clássico da cidade de Maringá, estado do Paraná. Como objetivos específicos, verificar, por meio de protocolos estabelecidos na área (EAT, BITE), o nível de propensão à BN e AN apresentada por adolescentes praticantes de ballet clássico com idade entre doze a dezoito anos, além de identificar a etiologia proposta para os transtornos alimentares nestas adolescentes.

\section{REVISÃO DE LITERATURA}

\subsection{Adolescentes}

O período de tempo que constitui o que se conhece como "adolescência" é afetado tanto pela biologia quanto pela cultura. Ela inclui pessoas com idade entre dez e vinte anos, sendo uma fase de intenso e rápido crescimento e desenvolvimento físico, psíquico e social, marcado por um período de aumentos acelerados tanto no peso quanto na altura, exigindo um aumento das necessidades nutricionais. ${ }^{3}$

Devido essas mudanças corporais, a maioria das adolescentes não fica satisfeita com as Conexões: revista da Faculdade de Educação Física da UNICAMP, Campinas, v. 11, n. 1, p. 171-187, jan../mar. 2013. 173 ISSN: $1983-9030$ 
formas que seu corpo adquire e acabam optando pela restrição de alimentos para poder reverter essa situação, buscando um padrão físico diferente do que possui geneticamente. ${ }^{4}$

O grau e os tipos de comprometimento do padrão alimentar normal variam muito. Alguns comportamentos inadequados (como exclusão de determinados tipos de alimentos ou de refeições ao longo do dia e realização de dietas não balanceadas) podem aparecer isoladamente ou fazer parte de um transtorno psiquiátrico. ${ }^{5-6}$

O aumento da prevalência dos transtornos psiquiátricos alimentares nesta etapa da vida, está associado ao padrão ideal de beleza imposto predominantemente ao sexo feminino. A cultura da magreza determina valores e normas que desencadeiam atitudes e comportamentos relacionados ao tamanho do corpo, à aparência e ao peso, ocorrendo principalmente durante o período de mudanças físicas. ${ }^{7}$

Por isso, muitas adolescentes buscam dietas instantâneas a fim de ficarem com os corpos desejados pelo o que a sociedade julga ser o certo, fazendo o que for preciso para alcançar esse padrão. Estima-se que os distúrbios alimentares afetam de 10 a $15 \%$ dos adolescentes, sendo $90 \%$ destes do sexo feminino. ${ }^{8}$

A prevalência de transtornos alimentares em adolescentes praticantes de ballet clássico é alta, pois além da grande preocupação com a imagem corporal, ainda há a exigência imposta pela própria modalidade. ${ }^{7}$

\subsection{Ballet Clássico}

O ballet clássico é um exercício físico caracterizado por movimentos de flexões e extensões, saltos e giros envolvendo velocidade e equilíbrio, além de requerer que o praticante possua graça, leveza e delicadeza. É denotado através do desenvolvimento de passos técnicos, harmoniosos e representações elaboradas. ${ }^{9}$

Trata-se de uma manifestação artística exigente no critério anatômico corporal, a qual o bailarino deve apresentar uma figura magra e longilínea, almejando um baixo percentual de gordura, resultando no criterioso cuidado no controle de peso. ${ }^{10}$ 
A prevalência dos transtornos alimentares é ainda mais elevada em pessoas que exercem determinadas atividades (modelos, bailarinas, atletas e profissionais da área da saúde). Estas populações provavelmente se tornam mais vulneráveis aos transtornos alimentares pelo fato de sofrerem, mais intensamente, pressões associadas a padrões estéticos e respondem por meio da exibição de um corpo magro a qualquer custo. ${ }^{11}$

O ballet com sua preocupação excessiva com a estética corporal, pode promover em seus praticantes transtornos alimentares como $\mathrm{AN}$ e BN. A busca do perfeccionismo, alta expectativa, pressão por parte dos instrutores e familiares e a instabilidade emocional podem levar aos transtornos alimentares. ${ }^{12}$

O ballet clássico é a transformação e o desenvolvimento da dança primitiva, que se baseava no instinto, em uma dança formada por passos diferentes, de ligações, de gestos e de figuras previamente elaborados para um ou mais participantes. ${ }^{13}$

A modalidade requer, além do belo, muito desempenho físico dos participantes. A prática da dança desenvolve sensibilidade, musicalidade, percepção, além das condutas psicomotoras coordenação, equilíbrio, tônus, lateralidade, noção espacial, noção temporal, ritmo, relaxamento e respiração. ${ }^{14}$

Desta forma, a imagem corporal acaba fazendo parte da rotina do ballet clássico. Sustentação, equilíbrio, sapatilhas de ponta e um corpo magro é uma constante na vida do bailarino, apesar de não se terem dados científicos de que para dançar seja necessário estar magro. ${ }^{1}$

Atualmente, o mundo em que se vive tem padrões de beleza cruéis que parecem essenciais à vida de muitas pessoas, principalmente aquelas que se encontram ligadas à área de dança.

As atletas e as bailarinas femininas enfrentam um grande risco para a AN. O sucesso atual no ballet, por exemplo, depende do desenvolvimento de um corpo flexível e extremamente esbelto. No atletismo, nos esportes de "aparência" (como ginástica e patinação artística), nos esportes de resistência (como corridas) e no fisiculturismo os riscos são ainda maiores. $^{15}$

Conexões: revista da Faculdade de Educação Física da UNICAMP, Campinas, v. 11, n. 1, p. 171-187, jan../mar. 2013.175 ISSN: 1983-9030 
As estimativas para os episódios dos transtornos do comer entre tais atletas estão tão altos como $60 \%$ a $70 \%$. Tais pessoas firmemente competitivas geralmente são perfeccionistas traço comum entre as pessoas com transtornos do comer. Esta luta pela melhora constante aparece em hábitos dietéticos assim como em seus desempenhos atléticos ou de dança. A anorexia também aparece na puberdade, fazendo que as atletas femininas jovens retenham uma forma muscular de menino, ou seja, sem o acúmulo normal de tecido adiposo subcutâneo nos seios e nos quadris o qual pode reduzir sua margem competitiva. ${ }^{15}$

Os treinadores e os professores freqüentemente multiplicam o problema ao encorajar um controle de calorias e perda do tecido adiposo e ao excessivo controle na vida dos atletas. Alguns chegam a ser até abusivos humilhando atletas que passam do limite de peso ou lhes impondo castigos.

\subsection{Transtornos Alimentares}

Transtornos alimentares são doenças caracterizadas por graves alterações do comportamento alimentar que podem ocasionar sérias agressões à saúde, principalmente quando se trata de crianças e adolescentes. A incidência dos transtornos praticamente dobrou nos últimos vinte anos, afetando predominantemente mulheres jovens. ${ }^{16}$

Os transtornos alimentares acometem especialmente jovens entre 12 a 25 anos e do sexo feminino, onde indivíduos que praticam alguma atividade ligada à manutenção da forma física estão mais predispostos para desenvolver esses quadros. ${ }^{17}$

Dentre os transtornos alimentares, o mais comum é a anorexia nervosa (AN) a qual é definida como sendo uma grave perturbação em relação ao controle patológico do peso corporal, onde o indivíduo apresenta distúrbios da percepção da forma corporal. ${ }^{18}$

Sabe-se que depois de vários episódios de AN, o indivíduo pode evoluir para uma possível bulimia nervosa $(\mathrm{BN})$ a qual apresenta uma prevalência média de 3 a $5 \%$ na população feminina adolescente e adulta jovem. Indivíduos com BN consomem grandes quantidades de alimentos, especialmente se submetido à situação de estresse, e, para evitar o ganho de peso, a culpa e a vergonha provocada pelo desconforto alimentar, apresentam Conexões: revista da Faculdade de Educação Física da UNICAMP, Campinas, v. 11, n. 1, p. 171-187, jan../mar. 2013.176 ISSN: 1983-9030 
comportamentos compensatórios tais como indução ao vômito. ${ }^{15}$

Embora existam fatores genéticos e ambientais ligados a esses distúrbios, existem traços de personalidade em comum entre aqueles que os desenvolvem tais como baixa alta-estima, sentimentos de impotência e medo da obesidade, de modo que os comportamentos alimentares parecem se desenvolver, como forma de controlar o estresse e a ansiedade. ${ }^{19}$

A etiologia desses transtornos alimentares é hoje concebida como multidimensional e inúmeros outros fatores parecem mediar o impacto da cultura no comportamento individual, entre eles a vulnerabilidade psicológica e biológica. ${ }^{1}$

\subsubsection{Anorexia}

AN é uma doença com riscos clínicos podendo levar à morte por desnutrição. É considerado um transtorno alimentar no qual a busca implacável por magreza leva a pessoa a recorrer a estratégias para perda de peso ocasionando importante emagrecimento. Os anoréxicos apresentam medo intenso de engordar mesmo estando extremamente magros. ${ }^{1}$

Em 95\% dos casos, acomete mulheres na faixa de treze a vinte anos podendo acontecer cada vez mais cedo. ${ }^{20}$

Durante o desenvolvimento da $\mathrm{AN}$, a perda de peso é retratada como um sinal de extraordinária proeza e autodisciplina, uma vez que o ganho de peso é percebido como uma inaceitável perda de autocontrole, este transtorno pode ser apresentado sob dois tipos principais. No primeiro, chamado tipo restrito, a perda do peso é obtida através de dietas ou jejum com ou sem exercícios excessivos. No segundo, chamado tipo compulsão periódica/purgativo, a ocorrência de episódios de compulsão alimentar associada a vômitos auto-induzidos, ao abuso de laxativos ou diuréticos é determinante. ${ }^{21}$

Na AN ocorre distorção do corpo, auto-negação e as pessoas se sentem e se vêem maiores do que realmente são mesmo quando se encontram abaixo do peso ou estando com um quadro de desnutrição. Este fato é denominado de distorção ou insatisfação da imagem corporal. $^{22}$

Adolescentes com enorme preocupação em relação ao peso e à forma física desenvolvem o 
desejo e a busca de se sentirem cada vez mais magros e, para isso, fazem da perda do peso o grande objetivo de vida. Restrições alimentares, prática de exercícios físicos em exagero e uso de medicações (laxativas, diuréticas ou inibidoras de apetite) são parte das estratégias. Porém, o insucesso pode predispor até a um quadro de dependência de drogas. $^{22}$

\subsubsection{Bulimia}

O termo BN foi introduzido por Gerald Russell em 1979. Originou-se do grego bous (boi) e limos (fome), referindo-se portanto, a uma fome tão grande quanto a de um boi, ou que pode levar alguém a comer um boi. Este termo acha-se descrito em fontes européias há mais de dois mil anos mantendo o seu significado, ou seja, um estado patológico de voracidade que leva à ingestão de uma quantidade exagerada de alimentos. ${ }^{23}$

Estudos ${ }^{24}$ mostram uma prevalência de 1 a $4 \%$ da população com maior acometimento no sexo feminino.

Habitualmente o início da doença é um pouco mais tardio do que na AN ocorrendo comumente no final da adolescência e no início da idade adulta, não havendo distinção de aparecimento na diferentes classes sociais. ${ }^{25}$

Esta se caracteriza por grande e rápida ingestão de alimentos com sensação de perda de controle, chamados episódios bulímicos, acompanhados de métodos compensatórios inadequados para o controle de peso, como vômitos auto-induzidos, uso de medicamentos (diuréticos, inibidores de apetite, laxantes), dietas e exercícios físicos extremos. ${ }^{25}$

As bulímicas apresentam história de insatisfação com o corpo, centram seus valores se baseando quase que exclusivamente em sua aparência cercando-se das mais variadas e extravagantes dietas. Apresentam sentimentos de baixa auto-estima e tendem com isto a se isolar socialmente, evitando reuniões sociais ou viagens. ${ }^{26}$

As adolescentes bulímicas tentam enfrentar o vazio interno com excesso de alimentos. Em busca de soluções idealizadas para tentar obter algum controle sobre si mesma, são compulsivas e sobrepõem o corpo emocional ao corpo biológico. Não comem em excesso 
por terem fome em excesso, mas buscando desesperadamente uma sobrevivência emocional, para se sentirem vivas. A anorexia e a bulimia não são coisas opostas: existem anoréxicas bulímicas e bulímicas que já foram anoréxicas. Trata-se de um ambíguo do vazio interno, e cada uma das pacientes busca preenchê-lo de forma diferente, porém complementar. $^{27}$

É evidente o acometimento psicológico nesta população de forma que o tratamento pode ser considerado mais complexo do que inicialmente suposto.

\section{METODOLOGIA}

Esta pesquisa se caracteriza como sendo do tipo descritivo a qual, visa descobrir e observar fenômenos existentes, situações presentes e eventos, procurando descrevê-los, classificálos, compará-los, interpretá-los e avaliá-los com objetivo e aclarar situações para idealizar futuros planos e decisões. ${ }^{28}$

A amostra compreendeu cinqüenta adolescentes do sexo feminino, com idades entre doze a dezoito anos, praticantes de ballet clássico de três academias de Maringá-PR. Os critérios para inclusão das participantes na pesquisa foram à faixa etária e ser do sexo feminino.

Foram utilizados para esta pesquisa dois questionários para avaliar a presença de sintomas relacionados a transtornos alimentares:

1. Teste de Atitudes Alimentares (EAT 26 - Eating Attitude Test) - instrumento que contém 26 perguntas sobre comportamento alimentar e imagem corporal. Trata-se de um questionário auto-aplicativo com 26 questões dirigidas à sintomatologia anoréxica, com as possibilidades de respostas: 1. Nunca; 2. Raramente; 3. Às vezes; 4. Freqüentemente; 5. Muito freqüentemente; 6. Sempre. A opção "6" vale 3 pontos, a opção "5" vale 2 pontos e a opção “4” vale 1 ponto, as demais $(1,2,3)$ não pontuam. Pontuações maiores que 20 $(\mathrm{EAT}+)$ são indicativas de sintomatologia relacionada à anorexia nervosa. ${ }^{29}$

2. Teste de Investigação Bulímica de Edimburgo (BITE - Bulimic Inventory Test of Edinburgh) - questionário auto-aplicativo com 30 questões para se determinar sintomatologia relacionada à bulimia nervosa, tendo como respostas "sim" ou "não". A Conexões: revista da Faculdade de Educação Física da UNICAMP, Campinas, v. 11, n. 1, p. 171-187, jan../mar. 2013. 179 ISSN: 1983-9030 
resposta "sim" representa a presença de sintoma, valendo 1 ponto e a resposta "não" representa ausência de sintoma, não pontuando. Porém nas questões 1, 13, 21, 23 e 31, pontua-se inversamente.

A escala BITE está dividida em duas subescalas: 1. Subescala de sintomas: aqueles que apresentam pontuação entre 10 e 19 são considerados com padrão alimentar não usual, entretanto, o avaliado não preenche todos os critérios para o diagnóstico de bulimia; aqueles com mais de 19 pontos consideram-se com padrão alimentar compulsivo, com grande possibilidade de apresentar bulimia nervosa. 2. Subescala de Severidade: mede a severidade do comportamento em função da sua frequiência. Os avaliados que apresentam pontuação igual ou superior a 5 têm um escore significativo, e os que apresentam uma pontuação maior do que 9 apresentam um alto grau de severidade. No BITE foi desconsiderada a subescala de severidade. ${ }^{30}$

A coleta de dados foi realizada pelos próprios pesquisadores antes das aulas de ballet de cada estabelecimento, por meio dos testes que avaliam a presença de sintomas relacionados aos transtornos alimentares. Foram escolhidos três locais na cidade de Maringá/PR que ofereciam esta modalidade de dança. Os questionários foram aplicados somente após liberação do estabelecimento e assinatura do termo de compromisso livre e esclarecido pelos sujeitos pesquisados.

Os dados foram analisados pela estatística descritiva por meio de porcentagens, gráficos e tabelas. Foi feito um teste de Qui-Quadrado para verificar a associação entre as academias e o tipo de transtorno em nível de $5 \%$ de erro.

\section{RESULTADOS}

$\mathrm{Na}$ Tabela 1 estão apresentados os resultados das avaliações realizadas com o teste de investigação bulímica para determinar sintomas relacionados à bulimia nervosa, onde se pode observar que apenas $2 \%$ da população avaliada apresentaram alto nível de incidência de bulimia nervosa com presença de comportamento alimentar compulsivo. 
Tabela 1-Análise da escala de sintomas relacionados à bulimia nervosa

\begin{tabular}{|c|c|c|c|c|}
\hline \multirow{3}{*}{ ACADEMIAS } & \multicolumn{3}{|c|}{ ESCALAS DE SINTOMAS } & \multirow{3}{*}{$\begin{array}{l}\text { TOTAL DA AMOSTRA } \\
(\mathbf{n = 5 0 )}\end{array}$} \\
\hline & ALTO & MÉDIO & BAIXO & \\
\hline & F $\%$ & F $\%$ & $\mathbf{F}$ & \\
\hline $\mathbf{A}$ & $1(2 \%)$ & $2(4 \%)$ & $7(14 \%)$ & 10 \\
\hline B & - & $4(8 \%)$ & $21(42 \%)$ & 25 \\
\hline $\mathbf{C}$ & - & $3(6 \%)$ & $12(24 \%)$ & 15 \\
\hline TOTAL & $1(2 \%)$ & $9(18 \%)$ & $40(80 \%)$ & $\mathrm{n}=50$ \\
\hline
\end{tabular}

Na Tabela 2 estão apresentados os resultados do teste de atitudes alimentares, EAT-26, no qual existem 6 tipos de respostas pontuadas numa escala de 0 a 3 pontos. Pontuação igual ou maior que 20 são indicativas de sintomatologia relacionada à anorexia nervosa e menor que 20 apresentam ausência de transtorno alimentar.

Tabela 2 - Porcentagem de bailarinas segundo o teste de atitudes alimentares EAT-26

\begin{tabular}{|c|c|c|c|c|c|}
\hline \multirow{3}{*}{ ACADEMIAS } & \multicolumn{4}{|c|}{ PONTUAÇÃO } & \multirow{3}{*}{$\begin{array}{c}\text { TOTAL DA AMOSTRA } \\
(\mathbf{n}=\mathbf{5 0})\end{array}$} \\
\hline & \multicolumn{2}{|c|}{$\geq 20$} & \multicolumn{2}{|c|}{$\leq \mathbf{2 0}$} & \\
\hline & $\mathbf{F}$ & $\%$ & $\mathbf{F}$ & $\%$ & \\
\hline $\mathbf{A}$ & 2 & $(4 \%)$ & 8 & $(16 \%)$ & 10 \\
\hline B & 8 & $(16 \%)$ & 17 & $(34 \%)$ & 25 \\
\hline $\mathbf{C}$ & 10 & $(20 \%)$ & 5 & $(10 \%)$ & 15 \\
\hline TOTAL & 20 & $(40 \%)$ & 30 & $(60 \%)$ & $\mathrm{n}=50$ \\
\hline
\end{tabular}

\section{DISCUSSÃO}

Padrão alimentar não usual foi apresentado por $18 \%$ da população avaliada (constatado através da escala média). Nesta população não existem critérios suficientes para a bulimia nervosa, porém o comportamento alimentar é não convencional. 
Já $80 \%$ da população avaliada apresentaram nível baixo de incidência de sintomas de BN encontrando-se dentro dos limites de anormalidade. Todavia, não houve associação significativa $(p=0,3687)$ entre academia e escala de sintomas, mostrando que a academia não interfere no grau de bulimia.

Tais resultados não estão em concordância com a literatura atual a qual afirma que a dança influencia diretamente a imagem corporal no sentido de buscar o emagrecimento a qualquer custo.

Os achados em outro estudo ${ }^{31}$, por exemplo, enfatizam que certos atletas, particularmente aqueles das práticas desportivas que enfatizam a magreza, como o ballet, correm o risco de desenvolverem transtornos alimentares.

Dados obtidos em pesquisas ${ }^{32}$ demonstram uma prevalência de transtornos alimentares entre atletas do sexo feminino em $42 \%$ para esportes estéticos (ginástica artística, GRD, ballet, patinação e etc.), $24 \%$ em esportes de endurance (corridas de fundo, triathlon e etc.), $17 \%$ em esportes técnicos e $16 \%$ em esportes com bola (vôlei, handebol, basquete e etc).

Talvez, esta discrepância possa ser explicada pelo fato das adolescentes apresentarem medo de responder a verdade absoluta em cada item questionado de forma que parte da verdade possa ter sido mascarada.

Verificou-se uma associação significativa $(\mathrm{p}=0,033)$ entre a academia e a pontuação no EAT-26. De forma geral, a análise da Figura 1 evidenciou que, da amostra total $(n=50)$, na academia A, 4\% das alunas apresentou resultado maior que 20 pontos e $16 \%$ apresentaram resultado menor que 20 pontos.

$\mathrm{Na}$ academia $\mathrm{B}, 16 \%$ apresentaram resultado maior que 20 pontos e $34 \%$ menor que 20 pontos. Já na academia C, $20 \%$ apresentaram resultados maiores que 20 pontos e $10 \%$ menor que 20 pontos.

Resultados similares foram observados em estudo ${ }^{32}$ realizado na Cidade do Cabo, na África Conexões: revista da Faculdade de Educação Física da UNICAMP, Campinas, v. 11, n. 1, p. 171-187, jan../mar. 2013.182 ISSN: 1983-9030 
do Sul, no qual examinaram sintomatologia referente à $\mathrm{AN}$ em quarenta e nove estudantes de Ballet do sexo feminino.

Todas as estudantes responderam o Eating Attitudes Test (EAT) e foi realizada entrevista semi-estruturada (Morgan-Russel Scales) com aquelas que pontuaram trinta ou mais no teste e/ou apresentaram amenorréia secundária ou primária, se tivessem dezesseis anos ou menos. Foi diagnosticada AN em apenas duas dançarinas $(4,1 \%)$.

Pesquisadores $^{33}$ documentaram os casos de duas bailarinas, uma com quinze e outra com dezesseis anos de idade, que cometeram tentativas de suicídio na cidade de Nantes, na França. Em ambas foi diagnosticada AN, associadas a transtornos mentais (depressão e comportamento histérico).

Dentre os métodos utilizados pela população avaliada para perda de peso destaca-se o abuso de laxantes e a indução ao vômito. O uso abusivo de laxantes foi o mais citado totalizando quatorze bailarinas e a indução ao vômito é praticada por sete bailarinas. ${ }^{34}$

No geral, a prevalência de transtornos alimentares nas bailarinas deste estudo foi de $12 \%$ o dobro da encontrada em estudantes na mesma faixa etária. Além disso, pontuações maiores que 30 no EAT se associaram mais a comportamentos bulímicos do que anoréxicos.

\section{CONCLUSÃO}

Mediante a análise dos resultados obtidos e com base no referencial teórico consultado, o estudo demonstrou que, em relação à bulimia nervosa, nenhuma das academias pesquisadas apresentou alto risco de desenvolvimento deste transtorno considerando os sintomas referidos.

Em relação à anorexia nervosa, um valor alto foi verificado em uma das academias pesquisadas (academia C) observando-se a prevalência de sintomatologia anoréxica. Desta forma, conclui-se que a prevalência de AN e BN em adolescentes do sexo feminino praticantes de ballet clássico da cidade de Maringá-PR não é tão expressiva como a literatura cita.

Conexões: revista da Faculdade de Educação Física da UNICAMP, Campinas, v. 11, n. 1, p. 171-187, jan../mar. 2013. 183 ISSN: 1983-9030 
Demais estudos, com uma amostra maior e mais significativa podem ser realizados a fim de resultados mais significativos e concretos.

Contudo, podemos citar que o papel da equipe multiprofissional que trabalha com esta população é acompanhar atentamente os hábitos alimentares, físicos e psicológicos daqueles com comportamento de risco e auxiliá-los na obtenção de melhor qualidade de vida.

\section{REFERÊNCIAS}

${ }^{1}$ SIMAS, J. P. N.; GUIMARÃES, A. C. A. Ballet Clássico e transtornos alimentares. Revista da Educação Física/UEM, Maringá, v.13, n.2, p.119-126, 2002.

${ }^{2}$ APPOLINARIO, J. C.; CLAUDINO, A. M. Transtornos alimentares. Revista Brasileira de Psiquiatria, São Paulo, v. 22, p. 28-31, 2000.

${ }^{3}$ GALlaHUE, D. L; OZMUN, I. C. Compreendendo o desenvolvimento motor: bebês, crianças, adolescentes e adultos. São Paulo: Phorte, 2001.

${ }^{4}$ JACOBSON M. S.; EISENSTEIN G.; COELHO, S. C. Aspectos nutricionais na adolescência. Adolescência Latinoamericana, Porto Alegre, v. 1, n. 2, p. 75-83, 1998.

${ }^{5}$ CORDAS, T.; NEGRÃO, A. Anorexia nervosa. In: ASSUMPÇÃO, F. B. (Ed.). Psquiatria da infância e adolescência. São Paulo: Santos, 1994. p. 283-290.

${ }^{6}$ FLEITLICH, B. W. et al. Anorexia nervosa na adolescência. Jornal de Pediatria, Rio de Janeiro, v. 76, n. 3, p. 323-329, 2000.

${ }^{7}$ NUNES, M. A. et al. Influencia da percepção do peso e do índice de massa corporal nos comportamentos alimentares anormais. Revista Brasileira Psiquiatria, São Paulo, v. 2, n.1, p. 21-27, 2001.

${ }^{8}$ CHIODINI, J. S.; OLIVEIRA. M. R. M. Comportamento alimentar de adolescentes: Conexões: revista da Faculdade de Educação Física da UNICAMP, Campinas, v. 11, n. 1, p. 171-187, jan../mar. 2013. 184 ISSN: $1983-9030$ 
aplicação de EAT-26 em uma escola publica. Saúde em Revista, Piracicaba, v. 5, n. 9, p. 53-58. 2003.

${ }^{10}$ BETANCOURT, H. et al. Composición corporal de bailarines élites de la compañía Ballet Nacional de Cuba. Revista Cubana de Alimentación y Nutricion, Habana, v. 17, n. 1, p. 8-22, 2007.

${ }^{11}$ FIATES, G. M. R, SALLES, R. K. Fatores de risco para o desenvolvimento de distúrbios alimentares: um estudo em universitárias. Revista de Nutrição, Campinas, v. 14, p.3-6, 2001.

${ }^{12}$ SCHAFLE, M. D. Segredos em medicina desportiva: respostas necessárias ao dia-a-dia em centros de treinamento, na clínica em exames orais e escritos. Porto Alegre: Artes Médicas, 1996.

${ }^{13}$ DI DONATO, S. Historia da dança. Revista Dançar, Rio de Janeiro, v.1, p.10, 1994.

${ }^{14}$ BAMBIRRA, W. Dançar e sonhar: a didática do ballet infantil. Belo Horizonte: Del Rey, 1993.

${ }^{15}$ BOSI, M. L. M.; OLIVEIRA, F. P. Comportamento bulímico em atletas adolescentes corredores de fundo. Revista Brasileira de Psiquiatria, São Paulo, v. 26, p. 32-34, 2004.

${ }^{16}$ PINZON, V.; NOGUEIRA, F. C. Epidemologia, curso e evolução dos transtornos alimentares. Revista de Psiquiatria, v. 31, n. 4, p.158-160, 2004.

${ }^{17}$ SOUZA, A.A et al. Estudo sobre anorexia e bulimia nervosa em universitárias. Psicologia: teoria e pesquisa, v. 27, n. 2, p.195-198, 2011.

${ }^{18}$ SAIKALI, C. J. et al. Imagem corporal nos transtornos alimentares. Revista de Psquiatria Clinica, São Paulo, v. 21, 2004.

${ }^{19}$ DUNKER, L. L. K.; PHILIPPI, T. S. Hábitos e comportamentos alimentares de Conexões: revista da Faculdade de Educação Física da UNICAMP, Campinas, v. 11, n. 1, p. 171-187, jan../mar. 2013.185 ISSN: 1983-9030 
adolescentes com sintomas de anorexia nervosa. Revista de Nutrição, Campinas, v.16, n.1, 2003.

${ }^{20}$ SEGATTO, C.; PADILlA, I.; FRUTUOSO, S. Por dentro da mente de uma anoréxica. Época, Rio de Janeiro, p. 55, nov. 2006.

${ }^{21}$ MAHAN, L. K.; STUMP, S. E. Alimentos, nutrição e dietoterapia. São Paulo: Roca, 2002.

${ }^{22}$ BUSSE, R. S. Anorexia bulimia e obesidade. São Paulo: Manole, 2004.

${ }^{23}$ PARRY-JONES, B. Historical terminology of eating disorders. Psychological medicine, Cambridge, v. 21, 1991.

${ }^{24}$ GARFINKEL, P. E.; GOLDBLOOM, D. S. Transtornos alimentares: anorexia nervosa e bulimia nervosa. In: CARLSON, G. A.; WELLER, E. B.; GARFINKEL, B. D. Transtornos psiquiátricos na infância e adolescência. Porto Alegre: Artes Médicas, 1992.

${ }^{25}$ ALVARENGA, M.; PHILIPPI, T. S. Transtornos alimentares: uma visão nutricional. São Paulo: Manole, 2004.

${ }^{26}$ FAIRBURN, C. G. The heterogenetty of bulimias nervosa and its implications for treatment. Journal of Psychossomatic Research, v. 35, p. 3-9, 1991.

${ }^{27}$ HERSCOVICI, C. A escravidão das dietas. Porto Alegre: Artes Médicas, 1997.

${ }^{28}$ MARTINS JUNIOR, J. Como escrever trabalhos de conclusão de curso: instruções para planejar e montar, desenvolver, concluir, redigir e apresentar trabalhos monográficos e artigos. 2. ed. Petrópolis: Vozes, 2008.

${ }^{29}$ GARNER D. M.; GARFINKEL, P. E. The eating attitudes tests: an index of symptoms of anorexia nervosa. Psychological Medicine, Cambridge, 1979. 
${ }^{30}$ HENDERSON, M.; FREEMAN, C. P. L. A self-rating scale for bulimia: the BITE. British Journal of Psychiatry, London, v. 150, p.18-24, 1987.

${ }^{31}$ GARNER, D. M. et al. Eating disorders among athletes: research and recommendations. Child Adolescent Psychiatry Clinical in North America, v. 7, n. 4, p. 839-857, 1998.

${ }^{32}$ GRANGE, D.; TIBBS, J.; NOAKES, T. D. Implications of a diagnosis of anorexia nervosa in a ballet school. International Journal of Eating Disorders, New York, v. 5, n. 4, 1994.

${ }^{33}$ SANCHEZ-CARDENAS, M.; PORTES, C. Eating disorders and suicide attempts in 2 dance students. Archives Pediatric, v. 3, n.1, p. 51-54, 1996.

${ }^{34}$ ABRAHAM, S. Eating and weight controlling behaviours of young ballet dancers. Psychopathology, Basel, v. 29, n. 4, p. 218-222, 1996.

Recebido em: 21 jun. 2012

Aceito em: 10 dez. 2012

Contato: Daniel Vicentini de Oliveira d.vicentini@hotmail.com 